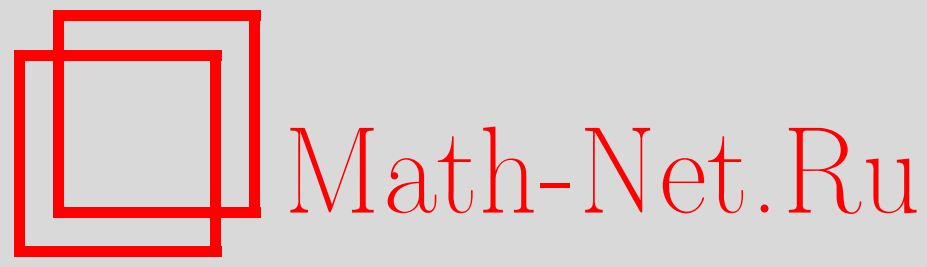

А. В. Романов, Ординарные полукаскады и их эргодические свойства, Функи. анализ и его прил., 2013, том 47, выпуск 2, 92-96

DOI: https://doi.org/10.4213/faa3115

Использование Общероссийского математического портала MathNet.Ru подразумевает, что вы прочитали и согласны с пользовательским соглашением

http://www . mathnet.ru/rus/agreement

Параметры загрузки:

IP : 54.164 .48 .24

26 апреля 2023 г., 15:18:53

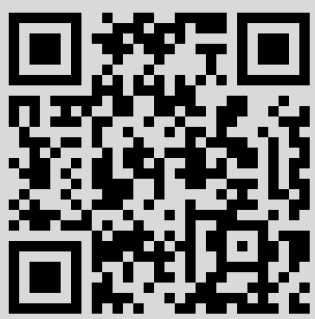


Зап. научн. сем. ПОМИ, 333 (2006), 62-65. [6] С. В. Кисляков, Н. Г. Сидоренко, Сибирск. матем. ж., 29:3 (1988), 64-77. [7] A. Pełczyński, K. Senator, Studia Math., 84:2 (1986), 169-215. [8] В. А. Солонников, Зап. научн. сем. ЛОМИ, 27 (1972), 194-210.

С.-Петербургское отделение Математического института им. В. А. Стеклова

Поступило в редакцию e-mail: skis@pdmi.ras.ru

1 августа 2012 г.

С.-Петербургский Государственный

Политехнический Университет

e-mail: dimax239@bk.ru

Исследовательская лаборатория им. П. Л. Чебышева

СПБГУ, Санкт-Петербург

e-mail: dms239@mail.ru

УДК 517.98

\section{Ординарные полукаскады и их эргодические свойства}

(C) 2013. A. B. Романов

Введение. В этой статье эргодические свойства дискретной динамической системы $(\Omega, \varphi)$, порожденной непрерывным преобразованием метрического компакта $\Omega$, формулируются в терминах трех ассоциированных с $(\Omega, \varphi)$ алгебротопологических объектов. Речь идет об описанных в [1], [2] обволакивающей полугруппе Эллиса $E(\Omega, \varphi)$ и операторной обволакивающей полугруппе Кёлер $\Gamma(\Omega, \varphi)$, а также, о рассмотренной в предшествующей работе автора [3] и связанной с $\Gamma(\Omega, \varphi)$ операторной полугруппе $G(\Omega, \varphi)$. Помимо этого изучаются представляющие самостоятельный интерес зависимости между тремя данными полугруппами. Как показано в [3], топологические характеристики компактных в соответствующих топологиях полугрупп $E(\Omega, \varphi), \Gamma(\Omega, \varphi)$ и $G(\Omega, \varphi)$ тесно связаны с эргодическими свойствами полукаскада $(\Omega, \varphi)$. Оказывается, подобного рода связи проявляются особенно ярко для класса дискретных динамических систем, обладающих метризуемой обволакивающей полугруппой $E(\Omega, \varphi)$ и называемых здесь ординарными. Ряд результатов о полугруппах Эллиса подобных систем (причем не только дискретных) можно найти в обзоре [4]. Известно, что ординарными оказываются все слабо почти периодические динамические системы.

Здесь предложено (теорема 4) альтернативное определение ординарности полукаскада $(\Omega, \varphi)$, состоящее в требовании метризуемости индуцированной полугруппы $G(\Omega, \varphi)$. Рассматривается также первоначально введенный Кёлер [2] и изученный в работах [4]-[6] класс так называемых ручных динамических систем $(\Omega, \varphi)$. В разд. 2 представлена иерархическая сводка известных ранее и некоторых новых свойств полукаскада $(\Omega, \varphi)$, формулируемых большей частью в терминах полугрупп $E(\Omega, \varphi), \Gamma(\Omega, \varphi)$ и $G(\Omega, \varphi)$.

В то же время главные результаты заметки относятся к слабой* сходимости операторных эргодических средних для ординарных и ручных полукаскадов. Сама идея рассмотрения подобной сходимости в эргодической теории восходит по существу к классической работе Крылова и Боголюбова [7]. 
1. Предварительные сведения. Пусть $\varphi-$ непрерывное (не обязательно обратимое) преобразование метрического компакта $\Omega$ и $(\Omega, \varphi)$ - соответствующий полукаскад на $\Omega$. Отображение $\varphi: \Omega \rightarrow \Omega$ порождает линейные сдвиги $U$ и $V=U^{*}$ в пространстве $X=C(\Omega)$ непрерывных скалярных функций на $\Omega$ и в сопряженном пространстве $X^{*}$ борелевских мер на $\Omega$. Обозначим через End $X^{*}$ нормированное пространство ограниченных линейных операторов в $X^{*}$. Обволакивающая полугруппа $E(\Omega, \varphi)$ полукаскада $(\Omega, \varphi)$ получается [1] замыканием семейства $\left\{\varphi^{n}, n \geqslant 0\right\}$ в отделимой топологии поточечной сходимости пространства всевозможных отображений $\Omega \rightarrow \Omega$. Заметим, что хотя в работе [1] рассматриваются обратимые динамические системы, большинство ее результатов справедливо также и в необратимом случае. Полугруппа $\Gamma(\Omega, \varphi)$ представляет собой [2] замыкание множества $\Gamma_{0}=\left\{V^{n}, n \geqslant 0\right\}$ в слабой* операторной топологии $W^{*} O$ пространства операторов End $X^{*}$. Полугруппа операторов $G(\Omega, \varphi)$ определяется [3] как $W^{*} O$-замкнутая выпуклая оболочка множества $\Gamma_{0}$. Множества $E(\Omega, \varphi), \Gamma(\Omega, \varphi)$ и $G(\Omega, \varphi)$ компактны в указанных топологиях. Если $A=A(\Omega)$ и $K=K(\Omega)$ - компактные в $w^{*}$-топологии пространства $X^{*}=C^{*}(\Omega)$ множества вероятностных борелевских мер и мер Дирака $\delta(\omega)$ на $\Omega$, то полугруппу $G(\Omega, \varphi)$ можно интерпретировать как обволакивающую полугруппу действия $W \times A \stackrel{V}{\longrightarrow} A$ абелевой полугруппы $W$ неотрицательных финитных числовых последовательностей с единичной суммой и со сверткой в качестве умножения. При этом $V \delta(\omega)=\delta(\varphi \omega), \omega \in \Omega$, так что $P K \subset K$ для всех операторов $P \in \Gamma(\Omega, \varphi)$. Соответствие $V \rightarrow \varphi$ индуцирует непрерывный алгебраический гомоморфизм $\pi: P \rightarrow p$ полугруппы $\Gamma(\Omega, \varphi)$ в полугруппу $E(\Omega, \varphi)$, действие которого понятным образом определяется из соотношения

$$
P \delta(\omega)=\delta(p \omega), \quad \omega \in \Omega,
$$

и оказывается эпиморфизмом.

Определение 1. Полукаскад $(\Omega, \varphi)$ называется ординарным, если его обволакивающая полугруппа $E(\Omega, \varphi)$ метризуема.

Определение 2 (см. [4]). Скажем, что динамическая система $(\Omega, \varphi)$ не хаотична, если каждое замкнутое полуинвариантное $(\varphi \Theta \subset \Theta)$ множество $\Theta \subset \Omega$ содержит траекторию $o(\omega), \omega \in \Theta$, устойчивую по Ляпунову относительно полукаскада $(\Theta, \varphi)$.

Здесь и далее $o(\omega)=\left\{\varphi^{n} \omega, n \geqslant 0\right\}$ для $\omega \in \Omega$. Понятие ручной динамической системы изначально вводилось [2] следующим образом.

Определение 3. Полукаскад $(\Omega, \varphi)$ называем ручным, если для любой непрерывной функции $x \in X$ и произвольной подпоследовательности $\{n(k)\}$ натурального ряда справедливо соотношение

$$
\inf _{a}\left\|\sum_{k=1}^{\infty} a_{k} x_{n(k)}\right\|_{X}=0,
$$

где $x_{m}(\omega)=x\left(\varphi^{m} \omega\right)(\omega \in \Omega)$ и нижняя грань берется по финитным вещественным последовательностям $a=\left\{a_{k}\right\}$ с $\sum_{k=1}^{\infty}\left|a_{k}\right|=1$.

Обозначим через $\Pi_{1}$ совокупность преобразований компакта $\Omega$, принадлежащих первому из расширяющихся классов Бэра, а через $\Pi_{b}$ множество всех борелевских отображений $p: \Omega \rightarrow \Omega$. 
Пусть $G_{0}-$ выпуклая оболочка множества $\Gamma_{0}$ в End $X^{*}$. Сеть операторов $T_{\alpha} \in G_{0}$ называется эргодической, если $T_{\alpha}(I-V) \stackrel{W^{*} O}{\longrightarrow} 0, I=$ id. Соответственно определяются эргодические последовательности операторов $T_{n} \in G_{0}$. Последовательность средних Чезаро $V_{n}=\frac{1}{n}\left(I+V+\cdots+V^{n-1}\right)$ эргодична, так как $V_{n}(I-V)=n^{-1}\left(I-V^{n}\right),\left\|V^{n}\right\|=1$. Отметим, что $W^{*} O$-сходимость эргодических сетей операторов $T_{\alpha}$ равносильна сходимости для произвольных функций $x \in X$ эргодических средних $T_{\alpha}^{*} x \rightarrow z, z \in X^{* *}$, в $w^{*}$-топологии пространства $X^{* *}=C^{* *}(\Omega)$. Для эргодических последовательностей подобная сходимость эквивалентна поточечной сходимости на компакте $\Omega$, но в общем случае может быть сильнее.

2. Классификация свойств компактных полукаскадов. Рассмотрим следующие классы D1-D6 свойств полукаскада $(\Omega, \varphi)$, связанных, в основном, с рассматриваемой задачей о слабой* сходимости эргодических средних.

D1: (a1) компакт $E(\Omega, \varphi)$ метризуем;

(b1) система $(\Omega, \varphi)$ не хаотична;

(c1) компакт $G(\Omega, \varphi)$ метризуем.

D2: (а2) компакт $G(\Omega, \varphi)$ есть пространство Фреше-Урысона;

(b2) $\operatorname{card} G(\Omega, \varphi)=\aleph$.

D3: (а3) полукаскад $(\Omega, \varphi)$ представляет собой ручную систему;

(b3) компакт $E(\Omega, \varphi)$ есть пространство Фреше-Урысона;

(c3) $\operatorname{card} G(\Omega, \varphi) \leqslant \aleph ;$

(d3) $E(\Omega, \varphi) \subset \Pi_{1}$;

(e3) $E(\Omega, \varphi) \subset \Pi_{b}$.

D4: (а4) операторы $T \in G(\Omega, \varphi)$ определяются своими значениями на мерах Дирака $\delta \in K(\Omega)$.

D5: (а5) выпуклое множество операторов $G(\Omega, \varphi)$ есть симплекс Шоке.

D6: (a6) операторы $P \in \Gamma(\Omega, \varphi)$ определяются своими значениями на мерах Дирака; тивна;

(b6) функция $\pi: \Gamma(\Omega, \varphi) \rightarrow E(\Omega, \varphi)$, определенная формулой $(1)$, инъек-

(c6) ex $G=\Gamma$;

(d6) ex $\Gamma=\Gamma$.

Здесь $\Gamma=\Gamma(\Omega, \varphi), G=G(\Omega, \varphi)$ и ех $(\cdot)$ обозначает совокупность крайних точек для множеств в End $X^{*}$. Часть свойств динамической системы $(\Omega, \varphi)$, входящих в классы D1, D3 и D6, рассматривалась ранее в [4]-[6]. Хотя эти работы посвящены обратимым динамическим системам, переход к необратимому случаю оказывается в необходимых ситуациях чисто техническим. Свойства (c6), (d6) обсуждались в [3]. Топологический компакт обладает свойством ФрешеУрысона, если операция замыкания множеств в нем определяется на языке последовательностей. Свойство (а5) означает [8], что конус в пространстве End $X^{*}$ с основанием $G(\Omega, \varphi)$ является векторной структурой. Если выполнено условие (b6), то определенная соотношением (1) функция $\pi: \Gamma(\Omega, \varphi) \rightarrow E(\Omega, \varphi)$ представляет собой одновременно гомеоморфизм и алгебраический изоморфизм, что позволяет отождествить полугруппы $\Gamma(\Omega, \varphi)$ и $E(\Omega, \varphi)$.

Теорема 4. Свойства полукаскада $(\Omega, \varphi)$ внутри каждого из классов D1, D2, D3, D6 попарно эквивалентны.

Таким образом, любое из свойств (b1), (c1) можно принять за альтернативное определение ординарности, а каждое из свойств (b3), (c3), (d3) или (e3) 
вполне характеризует ручные системы $(\Omega, \varphi)$. Подчеркнем, что эквивалентности $(\mathrm{a} 1) \Longleftrightarrow(\mathrm{b} 1)$ и $(\mathrm{a} 3) \Longleftrightarrow(\mathrm{b} 3) \Longleftrightarrow(\mathrm{c} 3) \Longleftrightarrow(\mathrm{d} 3)$ установлены в [4]-[6], а $(\mathrm{b} 6) \Longleftrightarrow(\mathrm{c} 6) \Longleftrightarrow(\mathrm{d} 6)$ получены в [3]. $\operatorname{Kaк~видим,~полукаскад~}(\Omega, \varphi)$ является ручным точно тогда, когда его обволакивающая полугруппа $E(\Omega, \varphi)$ состоит из борелевских преобразований. Значит, если эта полугруппа содержит преобразования, не входящие в первый класс Бэра, то она содержит и преобразования, не являющиеся борелевскими.

Таким образом, можно говорить о классах $\mathscr{D} 1-\mathscr{D} 6$ полукаскадов $(\Omega, \varphi)$, обладающих любым из (эквивалентных) свойств, входящих соответственно в классы D1-D6. Известно (см. [4]), что класс $\mathscr{D} 3$ ручных динамических систем не совпадает с классом $\mathscr{D} 1$ ординарных систем.

Теорема 5. Справедливы включения $\mathscr{D} 1 \subset \mathscr{D} 2 \subset \mathscr{D} 3 \subset \mathscr{D} 6$ u $\mathscr{D} 1 \subset \mathscr{D} 4 \subset$ $\mathscr{D} 5 \subset \mathscr{D} 6$.

Итак, в ординарном случае выпуклое компактное подмножество $G(\Omega, \varphi)$ линейного пространства End $X^{*}$ является симплексом Шоке и любой оператор $T \in G(\Omega, \varphi)$ определяется своими значениями на мерах Дирака $\delta(\omega), \omega \in \Omega$. В случае ручного полукаскада $(\Omega, \varphi)$ совокупность крайних точек множества $G(\Omega, \varphi)$ совпадает с множеством $\Gamma(\Omega, \varphi)$. Импликация (a3) $\Longrightarrow(\mathrm{b} 6)$ была установлена еще в [2]. Класс ординарных полукаскадов $\mathscr{D} 1$ достаточно обширен, ибо состоит из всех дискретных компактных динамических систем, не хаотичных в смысле определения 2. С другой стороны, известен пример [4] минимального дистального каскада на двумерном торе, не входящего даже в наиболее широкий из рассмотренных выше класс $\mathscr{D} 6$.

\section{3. Эргодические свойства ординарных и ручных полукаскадов.} Сформулируем теперь утверждения, относящиеся к эргодическим свойствам полукаскадов указанных типов. Соответствующие доказательства основаны на построениях разд. 2 и результатах работы [3]. Сходимость эргодических операторных сетей $T_{\alpha}$ в пространстве End $X^{*}$ понимается в смысле $W^{*} O$-топологии. Обозначим через $\Lambda(\Omega)$ множество эргодических мер полукаскада $(\Omega, \varphi)$. Пару точек $\omega_{1}, \omega_{2} \in \Omega$ называем проксимальной, если $\inf _{n \geqslant 0} \rho\left(\varphi^{n} \omega_{1}, \varphi^{n} \omega_{2}\right)=0$ для заданной на компакте $\Omega$ метрики $\rho$. Пусть, далее, $L$ - ядро (пересечение всех двусторонних идеалов) полугруппы $G(\Omega, \varphi)$, состоящее из проекторов $Q \in G(\Omega, \varphi)$ со свойством $V Q=Q$.

Теорема 6. Если полукаскад $(\Omega, \varphi)$ ординарен, то справедливы следующие утверждения:

(i) Каждая эргодическая сеть операторов $T_{\alpha}$ содержит сходящуюся подпоследовательность $T_{\alpha(k)}$. Все эргодические сети операторов $T_{\alpha}$ сходятся тогда u только тогда, когда для любого $\omega \in \Omega$ замыкание траектории о $\omega)$ содержит в себе единственное минимальное множество. Последнее верно с заменой слова «сети»на «последовательности».

(ii) Найдется такая сходящаяся эргодическая последовательность операторов $T_{n}$, что для любого $\omega \in \Omega$ мерз $T_{n} \delta(\omega)$ слабо* сходятся в $X^{*} \kappa$ эргодической мере $\mu_{\omega} \in \Lambda(\Omega)$, или, другими словами, асимптотическое (относительно $\left.\left\{T_{n}\right\}\right)$ распределение всех траекторий динамической системы $(\Omega, \varphi)$ определяется эргодическими мерами.

(iii) Если отношение проксимальности на $\Omega$ транзитивно, то все эргодические сети операторов $T_{\alpha}$ сходятся. 
(iv) Для каждой эргодической сети операторов $T_{\alpha}$ из поточечной сходимости функииональной сети $T_{\alpha}^{*} x$ на компакте $\Omega$ для всех непрерывных функиий $x \in X$ cледует $W^{*} O$-сходимость $T_{\alpha} \rightarrow Q, Q \in L$.

На самом деле утверждения (i)-(iii) верны в классе полукаскадов $\mathscr{D} 2$, а для справедливости (iv) достаточно свойства (a4). Как показано в [3], для произвольного полукаскада $(\Omega, \varphi)$ сходимость всех эргодических операторных сетей равносильна условию $\operatorname{card} L=1$.

Теорема 7. В случае ручного полукаскада $(\Omega, \varphi)$ можно сказать следующее.

(i) Минимальный центр притяжения $Z(\Omega, \varphi)$ совпадает с замыканием объединения всех минимальных множеств.

(ii) Если замыкание любой траектории о $(\omega), \omega \in \Omega$, содержит единственное минимальное множество, то все эргодические последовательности операторов $T_{n}$ сходятся и носитель каждой эргодической меры $\mu \in \Lambda(\Omega)$ есть минимальное множество.

Напомним, что минимальный центр притяжения $Z(\Omega, \varphi)$ динамической системы $(\Omega, \varphi)$ определяется как замыкание объединения носителей всех эргодических мер. Кроме того, отметим в этой связи строгую эргодичность минимальных ручных систем, установленную не так давно в [4], [9], [10]. Подчеркнем, что изложенные результаты нетрудно обобщить на случай динамических систем с непрерывным временем.

\section{ЛИТЕРАТУРА}

[1] R. Ellis, Lectures on Topological Dynamics, Benjamin, New York, 1969. [2] A. Köhler, Proc. Roy. Irish Acad. Sect. A, 95:2 (1995), 179-191. [3] А. В. Романов, Изв. РАН. Сер. матем., 75:6 (2011), 79-98. [4] E. Glasner, Topology Appl., 154:11 (2007), 2344-2363. [5] E. Glasner, M. Megrelishvili, Colloq. Math., 104:2 (2006), 223-283. [6] E. Glasner, Colloq. Math., 105:2 (2006), 283-295. [7] N. Kryloff, N. Bogoliouboff, Ann. of Math., 38:1 (1937), 65-113. [8] Р. Фелпс, Лекции о теореме Шоке, Мир, М., 1968. [9] W. Huang, Ergodic Theory Dynam. Systems, 26:5 (2006), 1549-1567. [10] D. Kerr, H. Li, Math. Ann., 338:4 (2007), 869-926.

Московский институт электроники и математики Национальный исследовательский университет «Высшая школа экономики» e-mail: vitkar48@inbox.ru
Поступило в редакцию 31 октября 2011 г.

Заведующая редакцией и научный редактор Г. М. Цукерман

Подписано к печати 22.04.2013. Дата выхода в свет 20.05.2013. Формат 70×100/16 Печать цифровая. Усл. печ. л. 7,8 . Усл. кр.-отт. 1,5 тыс. Бум. л. 3,0

Уч.-изд. л. 8,0. Тираж 181 экз. Заказ 1177. Цена свободная

Учредитель: Российская академия наук

Адрес редакции: 117966 Москва, ГСП-1, ул. Губкина 8, комн. 624. Тел. 938-37-56

Издатель: Российская академия наук, Издательство «Наука»,

117997 Москва, Профсоюзная, ул. 90

Отпечатано в ППП «Типография «Наука», 121099 Москва, Шубинский пер., 6 